\title{
Sweeping Away the Monopole Problem
}

\author{
G. Dvali, ${ }^{1,2}$ Hong Liu, ${ }^{3}$ and Tanmay Vachaspati ${ }^{4}$ \\ ${ }^{1}$ TH-Division, CERN, CH-1211 Geneva 23, Switzerland \\ ${ }^{2}$ ICTP, POB 586, 34100, Trieste, Italy \\ ${ }^{3}$ Theoretical Physics, Imperial College, Prince Consort Road, SW7 2BZ London, United Kingdom \\ ${ }^{4}$ Physics Department, Case Western Reserve University, Cleveland, Ohio 44106-7079
}

(Received 9 October 1997)

\begin{abstract}
We propose a new solution to the cosmological monopole problem in which domain walls sweep away the magnetic monopoles and subsequently decay. The solution does not require extensive finetuning or model building; it works for the prototype SU(5) grand unification model. More generally, it shows that defect interactions can lead to "defect erasure" in phase transitions and that this can be relevant to early universe physics. [S0031-9007(98)05517-3]
\end{abstract}

PACS numbers: 98.80.Cq, 11.30.Er, 14.80.Hv

All known attempts to unify the fundamental forces of nature predict the existence of magnetic monopoles [1]. In fact, in a cosmological context, all such attempts predict the existence of too many magnetic monopoles [2]. This is the monopole problem.

The monopole problem has at least three known solutions. The first is the inflationary solution to the monopole problem [3] whereby the universe inflates and dilutes the monopole density to acceptable levels. The second is called the Langacker-Pi mechanism [4] in which monopoles and antimonopoles get connected by strings which draw them together, leading to annihilation. The third mechanism [5] relies on nonrestoration of the grand unified symmetry and so there never was a phase transition in which monopoles were produced.

Here we show that there is yet another mechanism for solving the magnetic monopole problem that is economical-does not require complicated particle physics model building - and does not suffer from fine-tuning of the kind encountered in generic inflationary models. Indeed, the simplest concrete realization of the model is none other than the prototype grand unified SU(5) model that first inspired the inflationary solution.

The basic idea of this new mechanism is quite simple. The phase transition that produces magnetic monopoles also produces domain walls. The domain walls move through space and sweep up all the monopoles. When a monopole encounters a wall, it unwinds and dissipates and, in this way, the walls sweep away the monopoles from the universe. The alert reader would have realized at once that a danger in this scheme is that the monopole problem may have been replaced by a domain wall problem. This would be true if the walls were stable. However, as we discuss below, the walls in this scheme can be unstable at a lower energy scale and hence collapse and go away. The reason for the instability is that the discrete symmetry responsible for the walls is chosen to be approximate, or, in another rendering of the scenario, instanton effects violate the $Z_{2}$ symmetry and destabilize the walls.
The constraints on the model come from requiring that domain walls do not dominate the universe but live long enough to solve the monopole problem. As we shall see, these constraints can be met without any severe fine-tuning.

Let us now describe a concrete realization of the scenario. We consider the SU(5) grand unified model with an adjoint, $\Phi$, (and a fundamental) scalar field. The Higgs potential for $\Phi$ is the standard [6]

$$
\begin{aligned}
V(\Phi)= & -\frac{1}{2} m^{2} \operatorname{Tr} \Phi^{2}+\frac{h}{4}\left(\operatorname{Tr} \Phi^{2}\right)^{2} \\
& +\frac{\lambda}{4} \operatorname{Tr} \Phi^{4}+\frac{\gamma}{3} m \operatorname{Tr} \Phi^{3},
\end{aligned}
$$

where $\gamma$ is the dimensionless parameter that characterizes the explicit violation of the $Z_{2}$ symmetry: $\Phi \rightarrow-\Phi$.

Consider the case $\gamma=0$, in which case the spontaneous symmetry breaking

$$
\mathrm{SU}(5) \times Z_{2} \rightarrow\left[\mathrm{SU}(3)_{c} \times \mathrm{SU}(2)_{L} \times \mathrm{U}(1)_{Y}\right] / Z_{6}
$$

occurs when $\Phi$ acquires a vacuum expectation value $(\mathrm{VEV}) \quad \Phi_{0}=v \operatorname{diag}(2,2,2,-3,-3) / \sqrt{30}$, where $v=$ $m / \sqrt{\lambda^{\prime}}$ with $\lambda^{\prime} \equiv h+7 \lambda / 30$. To pick out this direction for the VEV, we need the following constraints on the parameters in the Higgs potential: $\lambda>0, h>-7 \lambda / 30$ (i.e., $\lambda^{\prime}>0$ ). The VEV $\Phi=-\Phi_{0}$ also leads to the symmetry breaking in (1). The two discrete vacua $\Phi= \pm \Phi_{0}$ are degenerate due to the exact $Z_{2}$ symmetry.

If $\gamma$ is nonzero but small enough to lead to the symmetry breaking in (1), the potential will continue to have two discrete minima which will now be nondegenerate. (Our scheme should also work for domain walls interpolating between vacuua with different symmetries but, for simplicity, we do not consider this possibility.) These minima will survive as long as the cubic term in the potential is small compared to the other terms and so leads to an order of magnitude constraint on $\gamma$ : it should be less than the other coupling constants in the model. Denote the VEV of $\Phi$ in the lower energy minimum by $\Phi_{+}$and 
that in the higher energy minimum by $\Phi_{-}$. In the limit of vanishing $\gamma, \Phi_{ \pm} \rightarrow \pm \Phi_{0}$.

It is well known that the symmetry breaking (1) leads to magnetic monopoles and $Z_{2}$ domain walls. What is less appreciated is that, if $\gamma \neq 0$, the symmetry breaking still leads to the formation of cosmological domain walls that interpolate between the two nondegenerate discrete minima denoted by + and - . In a cosmological setting, if the $Z_{2}$ symmetry is exact $(\gamma=0)$, we know that regions of the + and - vacuua will percolate [7] and the domain wall network will consist of an infinite domain wall and (very few) small isolated walls [8,9]. When $\gamma \neq 0$, the universe relaxes into the + vacuum with higher probability than in the - vacuum leading to "biased" domain wall formation [10]. If we denote the probability of a spatial domain being in the + vacuum by $P_{+}$and that of being in the - vacuum by $P_{-}$, we have $P_{-}=P_{+} \exp \left[-\Delta F_{V} / T_{c}\right]$, where $\Delta F_{V}$ is the free energy difference between two domains of volume $V$ of the \pm vacuua, and $T_{c}$ is the freeze-out temperature of the domains during the phase transition [11]. The free energy difference is given by $\Delta F_{V} \simeq 2 \gamma m v^{3} V / 3 \sqrt{30}$. If $P_{-}$is not too small, the walls will still percolate. For example, if percolation on a cubic lattice is a good description of the phase transition, the walls will percolate if $P_{ \pm}>0.31$. Using $P_{-}=1-P_{+}$, and requiring wall percolation imposes a constraint, $\Delta F_{V}<0.8 T_{c}$, which translates into the constraint: $\gamma \lesssim 7 T_{c} / m V v^{3}$. For weakly first order and second order phase transitions, on dimensional grounds, $V \sim m^{-3}, T_{c} \sim m$ and for values of $h$ and $\lambda$ that are not too extreme, we have $v \sim m$. Hence the constraint on $\gamma$ is quite mild and no fine-tuning is needed to get infinite walls to be produced. For strongly first order transitions, $V$ can be as large as the horizon volume at the grand unified phase transition and the constraint on $\gamma$ is very strong. Here we will consider only the weakly first order or second order phase transition for which $\mathrm{Vm}^{3}$ is not too large.

The energy difference across the walls for $\gamma \neq 0$ implies a force that drives the walls into the - regions. For example, a straight infinite wall would be pressure driven so that the volume in the higher energy - vacuum gets smaller. However, in a cosmological setting and at early times, the force of tension, $\sigma / R$ (where $\sigma=$ $4 m^{3} / 3 \lambda^{\prime}$ is the energy per unit area of the wall and $R$ is the radius of curvature) can be large compared to the pressure difference, $\Delta F_{V} / V$, across the wall. The radius of curvature of the walls $R$ is of order $T_{c}^{-1}$ for second order phase transitions. Hence, the pressure contribution to the dynamics of the walls is subdominant provided

$$
\gamma \lesssim \frac{10 \sqrt{\lambda^{\prime}}}{R m},
$$

which gives $\gamma \lesssim 10 \sqrt{\lambda^{\prime}}$. With this constraint satisfied, the wall evolution is just as in the exact $Z_{2}$ symmetry case where there is no pressure difference. As the walls are two dimensional surfaces moving in three spatial dimensions and are infinite in extent with no spatial symmetries, they will sweep out the entire volume of the universe in a time $\tau \sim R / v_{w}$ since $R$ is also the interwall distance and where $v_{w} \sim c$ is the wall velocity.

As the walls sweep the universe, they also sweep up the monopoles. Now what happens when a monopole hits a domain wall? Here there are two parameter dependent possibilities that we must discuss separately. The first is when $\Phi=0$ and the other is when $\Phi \neq 0$ inside the wall. To see that both cases are possible, consider the Higgs potential when we restrict $\Phi$ to lie along the diagonal, $a \lambda_{3}+b \lambda_{8}+c \tau_{3}+v Y$, where $\lambda_{3}$ and $\lambda_{8}$ are matrices from the $\mathrm{SU}(3)_{c}$ Cartan subalgebra, $\tau_{3}$ is the weak isospin, and $Y$ is the hypercharge generator, all matrices being normalized to unity. Usually one assumes that since $\Phi \rightarrow-\Phi$ across the wall, we must have $\Phi=0$ at the center of the wall and the SU(5) must be restored on the wall. However, for a wide range of parameter space this is not the case because some other component(s) of $\Phi$ (which vanish in the vacuum) can pick up a VEV and break the gauge symmetry inside the wall in a different fashion. This can be simply understood by examining the linearized Schrödinger equation for small excitations $\epsilon=\epsilon_{0}(x) e^{-i \omega t}$ ( $\epsilon$ is either the $a, b$, or $c$ component) in the wall background

$$
\left(-\partial_{x}^{2}+\left\{-m^{2}+[\bar{v}(x)]^{2}(h+\lambda r)\right\}\right) \epsilon_{0}=\omega^{2} \epsilon_{0},
$$

where the wall is taken to lie in the $x=0$ plane and for $\epsilon$ being in the $a, b, c$ directions we have $r=2 / 5,2 / 5$, and $9 / 10$, respectively. The function $\bar{v}(x)$ is the profile of the wall which for a planar infinite wall can be approximated by a kink solution $\bar{v}(x)=v \tanh (m x / \sqrt{2})$. When $\lambda=12 \lambda^{\prime}$ and $r=2 / 5$, the Schrödinger equation is identical to the equation obtained when solving for perturbations around the kink solution [12]. Then there is a zero mode corresponding to the translation of the kink. From this we deduce that the domain wall will have $\Phi=0$ at the center only if $\lambda^{\prime}<\lambda / 12$ as there are no bound state (negative $\omega^{2}$ ) solutions to the Schrödinger equation if this condition holds.

We must comment here that even if one forgets the monopole problem, from the cosmological viewpoint one prefers the range of parameters for which $\Phi$ vanishes on the wall. The reason is that if $\Phi$ is nowhere zero in space, then the domain walls may never collapse even though the $Z_{2}$ is only approximate [13]. To see this, let $b$ be a component that is nonzero on the wall and define a complex number $\psi=\bar{v}+i b$ with the boundary conditions $\psi= \pm v$ on opposite sides of the wall. This configuration is equivalent to the winding of the phase of $\psi$ by $\Delta=\pi$ through the wall. Since the expectation value $|\psi|$ is nowhere zero, the winding of the phase is well defined and one can distinguish walls with $\Delta= \pm \pi$ when one crosses them in one direction. Now walls with opposite winding can annihilate each other and disappear, but pairs with the same winding cannot. So when two 
neighboring walls with equal winding are pushed towards each other due to the pressure differences, they cannot annihilate, but instead form a "bound state": a $\Delta=2 \pi$ domain wall [13]. Although not truly stable (these can decay via quantum nucleation of holes [14]) they can be stable for all practical purposes if the expectation value of the Higgs field in the core is large. This consideration disfavors (but does not exclude) the range of parameters for which $\Phi \neq 0$ inside the wall.

If $\Phi=0$ inside the wall, the full SU(5) symmetry is restored there. If, however, $\Phi \neq 0$ inside the wall, the symmetry inside the wall is not the full SU(5) but only a subgroup which is different from the unbroken subgroup in the vacuum. We expect that our mechanism will work as long as the symmetry inside the wall is large enough so that monopoles can unwind there. To keep the discussion simple, we will consider only the $\Phi=0$ case here.

The interaction of monopoles and domain walls has not been investigated in detail but there are strong indications that the monopoles will unwind on entering the wall where the full SU(5) symmetry is restored. These indications are as follows.

(i) There is an attractive force between the monopoles and the walls since monopoles can save the expense of having to go off the vacuum in their core by moving on to the wall. So the monopoles can form bound states with the walls. [We observe here that the domain wall and monopole bound state can lead to a classical realization of a D-brane if the $\mathrm{SU}(3)_{c}$ symmetry group further breaks to $Z_{3}$ since now the monopoles bound to the walls will be connected by strings. Related constructions may also be found in [15].]. Then, as there is no topological obstruction to the unwinding of monopoles on the wall, the monopoles on the wall can continuously relax into the vacuum state.

(ii) The investigation of a similar system-Skyrmions and walls - has been dealt with in full detail in Ref. [16]. These authors find that the Skyrmion hits the wall, sets up traveling waves on the wall, and dissipates. They also find that, even though it is topologically possible for the Skyrmion to penetrate and pass through the domain wall, this never happens. They attribute their finding to the coherence required for producing a Skyrmion. That is, the penetration of a Skyrmion may be viewed as the annihilation of the incoming Skyrmion on the wall and the subsequent creation of a Skyrmion on the other side. However, the annihilation results in traveling waves along the wall that carry off a bit of the coherence required to produce a Skyrmion on the other side. Hence, even though there is enough energy in the vicinity of the collision, a Skyrmion is unable to be created on the other side of the wall. We think that these considerations apply equally well to monopole-wall interactions and that monopoles will - for all cosmological purposes - never penetrate the wall.

(iii) Energetically, the most favored state is where the monopole unwinds. Even if this does not happen in a single monopole-wall collision, it will occur if there are several interactions. Given that the walls are very efficient at sweeping the universe, multiple monopolewall interactions can easily occur.

(iv) The interactions of vortices and domain walls separating the $A$ and $B$ phases of ${ }^{3} \mathrm{He}$ have been studied and also observed experimentally. It is found that singular vortices do not penetrate from the $B$ phase into the $A$ phase [17].

Based on the arguments above, we conjecture that the monopoles are trapped on the walls and since they can unwind on the wall, will spread out as traveling waves. Eventually, as we will see below, the percolated wall will collapse and all the monopoles will be eliminated. (This will be simplest to see in a closed universe in which the total magnetic charge must vanish.)

In view of the fact that we have not proved that monopoles will unwind, it is useful to derive constraints on the probability of unwinding that will enable a successful resolution of the monopole problem. Let $\xi(t)$ be the average wall separation at time $t$. Then the time for the wall to move a distance $\xi(t)$ is $\tau(t)=\xi(t) / v_{w}$. We will further assume that the wall network at time $t_{0}+\tau\left(t_{0}\right)$ is completely independent of the network at time $t_{0}$ and provides a "fresh" (uncorrelated) set of walls that sweep the universe. The number of "correlation times" between wall formation at time $t_{f}$ and wall decay at time $t_{d}$ is $N=\log _{2}\left[\left(t_{d}-t_{f}\right) / \tau\left(t_{f}\right)\right]$. Let $1-p$ denote the probability that a monopole will survive for a correlation time. (This includes both the possibility that the monopole may not encounter a wall and that it may not unwind after being hit by a wall.) We will assume that $p$ and $\alpha=t / \xi(t)$ are constants which is reasonable if the wall network scales. Then the probability that a monopole will survive until the time $t_{d}$ is $S=(1-p)^{N}$ and this is constrained by cosmology to be less than a critical value $S_{*} \sim 10^{-12}$. Hence we need $p>1-S_{*}^{1 / N}$. To derive a numerical estimate of the upper bound on $N$, we take $t_{d} \sim(G \sigma)^{-1}$ (the time at which walls would start dominating [1]) and $\tau\left(t_{f}\right) \sim M_{P} / v^{2}$, where $M_{P} \sim 10^{19} \mathrm{GeV}$ is the Plank scale. This gives $N \sim \log _{2}\left[M_{P} / \sqrt{\lambda^{\prime}} v\right]$. For $v / M_{P}=10^{-4}$ and $\lambda^{\prime}=0.1, N$ is about 15. Therefore, with these parameters, we need $p>0.8$ for a cosmologically acceptable number of monopoles to have survived.

After the monopoles have been swept up by the walls, we are left with walls that are continually straightening out [18]. At a certain time, the condition in Eq. (2) is violated, and the walls start collapsing due to the pressure difference between the two sides. The walls will decay before they come to dominate the universe provided [1] $\Delta F_{V}>G \sigma^{2} V$. This gives an additional constraint on $\gamma$ :

$$
\gamma>\frac{10 G \sigma \sqrt{\lambda^{\prime}}}{m} \sim \frac{10}{\sqrt{\lambda^{\prime}}}\left(\frac{M_{G}}{M_{P}}\right)^{2},
$$

where $M_{G} \sim 10^{14} \mathrm{GeV}$ is the grand unified theory (GUT) scale. Once the walls are pressure driven, the volume 
fraction in the $\Phi_{+}$vacuum will increase at the expense of the $\Phi_{-}$vacuum. Finally all of space will be in the $\Phi_{+}$ vacuum and no domain walls will remain.

Summarizing the strongest constraints on $\gamma$, we need

$$
10^{-9} \lambda^{\prime-1 / 2} \lesssim \gamma \lesssim 10 \lambda^{\prime+1 / 2}
$$

for the domain walls to sweep away the monopoles and subsequently decay safely. The lower bound comes from requiring that the walls never dominate the universe and the upper bound comes from requiring that the walls percolate and that there is a period during which the wall evolution is tension dominated. (If the walls do not percolate, they will all be finite and will collapse without sweeping through the whole volume of the universe. If the pressure term becomes important before the monopoles have been swept away, some of the monopoles that were formed in the $\Phi_{+}$vacuum will not be swept up by the walls and will survive. In these cases, depending on the fraction of space that remains unswept, we may or may not have a monopole problem.)

The domain walls in the $\gamma=0$ case can also be eliminated if the $Z_{2}$ symmetry is anomalous under a strongly coupled $\mathrm{SU}(N)$ gauge group [19]. This can be the case if $\Phi$ gives mass to an odd number of fermionic flavors transforming in the fundamental representation of $\mathrm{SU}(N)$. Then, if there is no extra matter charged under $\mathrm{SU}(N)$, the $Z_{2}$ symmetry is expected to be explicitly broken by instantons. Provided the strong scale of $\mathrm{SU}(N), \Lambda$, is smaller than $M_{G}$, and the Yukawa coupling constants of fermions are of order one, the instantoninduced energy difference between the \pm vacua is $\sim \Lambda^{4}$. This bias will turn on only at a time $t_{\text {anom }}=M_{P} / \Lambda^{2}$ and the requirement that walls never dominate the universe leads to the bound $\Lambda^{2}>M_{G}^{3} / M_{P}$. (This rules out QCD as the source for an explicit bias.)

Yet another source of bias [20] can be a higher dimensional gravity-induced Planck scale suppressed operators that, on general grounds, are expected not to respect the global symmetries of the theory.

This resolution of the monopole problem frees inflation from having to occur during or after the GUT phase transition. Our considerations also show that the interactions of various defects produced during a phase transition can be vital to cosmology. In particular, one class of defects can erase another class.

G. D. thanks Savas Dimopoulos for discussions. T. V. is grateful to Alan Guth, Rich Holman, Misha Shaposhnikov, Mark Trodden, and Alex Vilenkin for helpful comments, to Grisha Volovik for explaining the interactions of defects in ${ }^{3} \mathrm{He}$ and providing references, and, to the DoE for support.

[1] A. Vilenkin and E.P.S. Shellard, Cosmic Strings and Other Topological Defects (Cambridge University Press, Cambridge, England, 1994).

[2] J. Preskill, Phys. Rev. Lett. 43, 1365 (1979).

[3] A. H. Guth, Phys. Rev. D 23, 347 (1981); A. D. Linde, Particle Physics and Inflationary Cosmology (Harwood Academic, New York, 1990).

[4] P. Langacker and S.-Y. Pi, Phys. Rev. Lett. 45, 1 (1980).

[5] G. Dvali, A. Melfo, and G. Senjanovic, Phys. Rev. Lett. 75, 4559 (1995); see also P. Salomonson, B. S. Skagertan, and A. Stern, Phys. Lett. 151B, 243 (1985). For a solution in supersymmetry, see G. Dvali, L. M. Krauss, and H. Liu, hep-ph/9707456, CERN-CWRU Report No. CERN-TH/97-123/CWRU-P5-1997; G. Dvali and L. M. Krauss (to be published).

[6] T.P. Cheng and L.F. Li, Gauge Theory of Elementary Particle Physics (Oxford, London, 1984).

[7] D. Stauffer, Phys. Rep. 54, 1 (1979).

[8] J. A. Harvey, E. W. Kolb, D. B. Reiss, and S. Wolfram, Nucl. Phys. B201, 16 (1982).

[9] T. Vachaspati and A. Vilenkin, Phys. Rev. D 30, 2036 (1984).

[10] T. Vachaspati, Phys. Rev. D 44, 3723 (1991).

[11] W. Zurek, Phys. Rep. 276, 177 (1996).

[12] R. Rajaraman, Solitons and Instantons (North-Holland, Amsterdam, 1982).

[13] G. Dvali, Z. Tavartkiladze, and J. Nanobashvili, Phys. Lett. B 352, 214 (1994).

[14] A. Vilenkin and A. E. Everett, Phys. Rev. Lett. 48, 1867 (1982).

[15] S. M. Carroll and M. Trodden, CWRU-NSF Report No. CWRU-P16-97/NSF-ITP/97-117, 1997 (to be published).

[16] A. Kudryavtsev, B. Piette, and W. J. Zakrzewski, hep-th/ 9709187, DTP-97/25, 1997.

[17] H.-R. Trebin and R. Kutka, J. Phys. A 28, 2005 (1995); T. Sh. Misirpashaev, Sov. Phys. JETP 72, 973 (1991); M. Krusius, E. V. Thuneberg, and U. Parts, Physica (Amsterdam) 197B, 376 (1994).

[18] The cosmological evolution of domain walls has been discussed in W. H. Press, B. S. Ryden, and D. N. Spergel, Astrophys. J. 347, 590 (1989); M. Hindmarsh, Phys. Rev. Lett. 77, 4495 (1996); D. Coulson, Z. Lalak, and B. Ovrut, Phys. Rev. D 53, 4237 (1996); S. E. Larsson, S. Sarkar, and P. J. White, Phys. Rev. D 55, 5129 (1997).

[19] J. Preskill, S.P. Trivedi, F. Wilczek, and M. B. Wise, Nucl. Phys. B363, 207 (1991).

[20] B. Holdom, Phys. Rev. D 28, 1419 (1983); B. Rai and G. Senjanovic, Phys. Rev. D 49, 2729 (1994). 